\title{
THE SIGNIFICANCE OF VARICEAL HAEMORRHAGE IN GHANA: A RETROSPECTIVE REVIEW
}

\author{
T. N. A. ARCHAMPONG ${ }^{1,2}$, K. TACHI ${ }^{1,2}$, A. A. AGYEI ${ }^{1,2}$ and K. N. NKRUMAH ${ }^{1,2}$ \\ ${ }^{1}$ Department of Medicine and Therapeutics, University of Ghana School of Medicine and Dentistry, Box 4236, \\ Korle-bu, Accra, Ghana, ${ }^{2}$ Department of medicine and Therapeutics, Korle-Bu Teaching Hospital, Box 77, \\ Korle-Bu, Accra, Ghana
}

DOI: $h t t p: / / d x . d o i . o r g / 10.4314 / g m j . v 49 i 3.3$

Corresponding Author: Dr. Timothy N.A. Archampong

Email: tnaa@doctors.net.uk

Conflict of Interest: None declared

\section{SUMMARY}

Background: This study describes the burden of bleeding oesophageal varices at the main tertiary referral centre in Accra.

Design: Retrospective design to describe the endoscopic spectrum and review mortality data following acute upper gastro-intestinal bleeding at the Korle-Bu Teaching Hospital. Endoscopic data was reviewed in the Endoscopy Unit between 2007 and 2010. Mortality data was collated from the Department of Medicine between 2010 and 2013.

Interventions: The study questionnaire compiled clinical and demographic characteristics, endoscopic diagnoses, length of hospital admission and treatment regimens.

Main outcome measures: Aetiology and time-trend analysis of mortality rates following acute upper gastro-intestinal bleeding; variceal bleeding treatment modalities.

Results: On review of the endoscopic diagnoses, gastro-oesophageal varices were identified in $21.9 \%$ of cases followed by gastritis $21.7 \%$, duodenal ulcer, $17.0 \%$, and gastric ulcer, $13.2 \%$. Gastro-oesophageal varices were the predominant cause of death from acute upper gastro-intestinal haemorrhage from $46 \%$ in 2010 to $76 \%$ in 2013 . Outcomes following acute upper gastro-intestinal bleeding were dismal with some $38 \%$ of fatalities occurring within the first 24 hours. Injection sclerotherapy was the dominant endoscopic modality for secondary prevention of variceal bleeding in comparison with band ligation, mainly as a result of cost and availability.

Conclusions: At the tertiary centre in Accra, variceal bleeding is an increasingly common cause of acute upper gastro-intestinal haemorrhage in comparison with previous reviews in Ghana. Its significantly high in-hospital mortality reflects inadequate facilities to deal with this medical emergency. A strategic approach to care with endoscopic services equipped with all the necessary therapeutic interventions will be vital in improving the outcomes of variceal bleeding in Ghana.
Keywords: gastro-oesophageal; varices; bleeding; gastro-intestinal, Ghana.

\section{INTRODUCTION}

Acute upper gastro-intestinal bleeding (AUGIB) is defined as bleeding that results from lesions located above the ligament of Treitz, a thin suspensory muscle attached to the duodenum. ${ }^{1}$ It is a common reason for emergency referral to hospital in patients with gastrointestinal disease. ${ }^{1}$

AUGIB can be either variceal or non-variceal. ${ }^{2}$ Sources of non-variceal bleeding include peptic ulcer disease (PUD), oesophagitis, Mallory-Weiss tear, vascular anomalies, gastric and oesophageal malignancy. ${ }^{3}$ Gastro-oesophageal varices (GOV) are dilated tortuous anastomotic veins prominent at the lower oesophagus and gastro-esophageal junction and are the most clinically relevant porto-systemic collaterals in cirrhosis and portal hypertension. ${ }^{4}$

They develop from connections between the short gastric and coronary veins and the oesophageal, azygous and intercostals veins ${ }^{5}$. Varices will be present in $40 \%$ of patients with clinically compensated cirrhotic liver disease ${ }^{2}$. This increases to $60 \%$ when decompensated with $90 \%$ developing oesophageal varices during the course of the disease. ${ }^{4}$

Variceal bleeding is a life threatening complication of portal hypertension ${ }^{6}$. It is a major cause of morbidity and death in patients living with liver cirrhosis ${ }^{7}$ and accounts for $70 \%$ of all AUGIB episodes in the context of portal hypertension ${ }^{5}$. The risk of bleeding increases with increasing pressure and size of the varices as well as decreasing thickness of the variceal wall reflected by the red dot sign at endoscopy. ${ }^{3}$ 
Variceal haemorrhage carries a significant mortality of $20-30 \%$, therefore the identification and prophylactic treatment of patients at risk for esophageal bleeding is mandatory. ${ }^{8}$

This study describes the scope of the problem and the therapeutic options in Ghana, a country with a significant burden of liver cirrhosis. ${ }^{9}$

\section{METHODS}

This study utilized a retrospective design to describe the endoscopic spectrum of disease following upper gastro-intestinal (GI) endoscopy for AUGIB at the Endoscopy Unit, Korle-Bu Teaching Hospital (KBTH) between June, 2007 and August 2010. Additionally, it reviewed mortality data from AUGIB in the Department of Medicine, KBTH from January, 2010 to November 2013.

These time periods were studied because endoscopy and mortality data were consistently archived and available for analysis. The study was conducted between September 2012 and December 2013. Korle-Bu Teaching Hospital (KBTH) is the premier Teaching Hospital in the capital city of Ghana, Accra, with an estimated population of 3.9 million people. It is the main tertiary referral centre in Accra serving the majority of the southern half of Ghana.

The hospital has a total bed capacity of one thousand six hundred (1600) with a daily out-patient department (OPD) attendance of about one-thousand-two-hundred (1200) and an annual turnover of about three hundred and fifty thousand $(350,000)$ patients. The Endoscopy Unit runs two four-hour sessions per day on four days of the week. Approximately five patients attend each endoscopy session for upper-GI endoscopy.

Mortality data in the Department of Medicine, KBTH, was derived from monthly departmental records while endoscopic data was captured from the Endoscopy Unit Log-book. Departmental mortality records review captured demographic data, duration of illness, symptoms and subsequent diagnosis.

Endoscopically confirmed diagnoses of all mortality cases following AUGIB were recorded in this review. Where there was no prior documentation of endoscopic diagnosis, the aetiology was indicated as undetermined. Consenting patients with GOVs undergoing injection sclerotherapy during the study period were administered a structured questionnaire.

It included the sclerosant used, attendant complications such as pain, bleeding, dysphagia and endoscopic stric- ture formation. Data were analyzed using Microsoft Excel and SPSS 16. Categorical data were expressed as proportions and presented in tables and bar charts.

\section{RESULTS}

Six-hundred and ninety-five (695) cases of AUGIB were investigated at the Endoscopy Unit, KBTH between 2007 and 2010 presenting as haematemesis and/or melaena. Table 1 demonstrates the endoscopic diagnoses following upper GI endoscopy. On review of the common pathologies encountered, GOVs were identified in $21.9 \%$ of cases followed by gastritis $21.7 \%$, duodenal ulcer (DU) $17.0 \%$, and gastric ulcer (GU) $13.2 \%$.

Table 1 the aetiology of upper-GI haemorrhage following endoscopy at the Korle-Bu Teaching Hospital: $2007-2010,(n: 695)$

\begin{tabular}{|l|l|l|}
\hline Endoscopic diagnosis & N & $\mathbf{\%}$ \\
\hline Gastro-esophageal varices & 144 & 20.7 \\
\hline Intra-gastric varices & 8 & 1.2 \\
\hline Esophagitis & 41 & 5.9 \\
\hline Duodenal ulcer & 118 & 17.0 \\
\hline Gastric ulcer & 92 & 13.2 \\
\hline Gastric erosion & 27 & 3.9 \\
\hline Gastritis & 151 & 21.7 \\
\hline Gastric cancer & 40 & 5.8 \\
\hline Normal & 139 & 20.0 \\
\hline
\end{tabular}

Table 2 shows the mortality patterns following AUGIB at the KBTH's Department of Medicine. One-hundred and ninety-six (196) mortality cases from AUGIB were recorded between 2010 and 2013 of which $70.1 \%$ were male and 29.1\% female. AUGIB represented 3.8\% $(n=37)$ of all-cause mortalities in 2010, 4.3\% $(n=44)$, 2011 ; 6.7\% $(n=66), 2012$ and 5.8\% $(n=52), 2013$ respectively.

Table 2 Mortality data on acute upper GI haemorrhage in the Department of Medicine, KBTH

\begin{tabular}{|c|c|c|c|c|}
\hline & 2010 & 2011 & 2012 & 2013 \\
\hline Total Admissions & 6735 & 6801 & 7501 & 7114 \\
\hline Total all-cause mortality cases & 966 & 1014 & 981 & 890 \\
\hline $\begin{array}{l}\text { Total deaths from AUGIB: (n) } \\
\text { - Deaths from variceal bleed- } \\
\text { ing } \\
\text { - Deaths from bleeding PUD } \\
\text { (DU+GU) } \\
\text { - Deaths from AUGIB (unde- } \\
\text { termined aetiology) }\end{array}$ & $\begin{array}{l}37 \\
17(45.9 \%) \\
8(21.6 \%) \\
12(32.4 \%)\end{array}$ & $\begin{array}{l}44 \\
28(63.6 \%) \\
9(20.5 \%) \\
7(15.9 \%)\end{array}$ & $\begin{array}{l}66 \\
51(77.3 \%) \\
7,(10.6 \%) \\
8,(12.1 \%)\end{array}$ & $\begin{array}{l}52 \\
40(76.9 \% \\
4,(7.7 \%) \\
8,(15.4 \%\end{array}$ \\
\hline
\end{tabular}

Figure 1 illustrates the age distribution of mortality cases from AUGIB with the commonest age group being $31-40$ years, $(24.5 \%)$ and $41-50$ years, $(25 \%)$. Bleeding GOVs were the predominant cause of death from AUGIB ranging from $45.9 \%(n=17)$ in 2010 to $76.9 \%(n=40)$ in 2013 . In 2013, the GOV: PUD mortality ratio was as high as 10:1. 


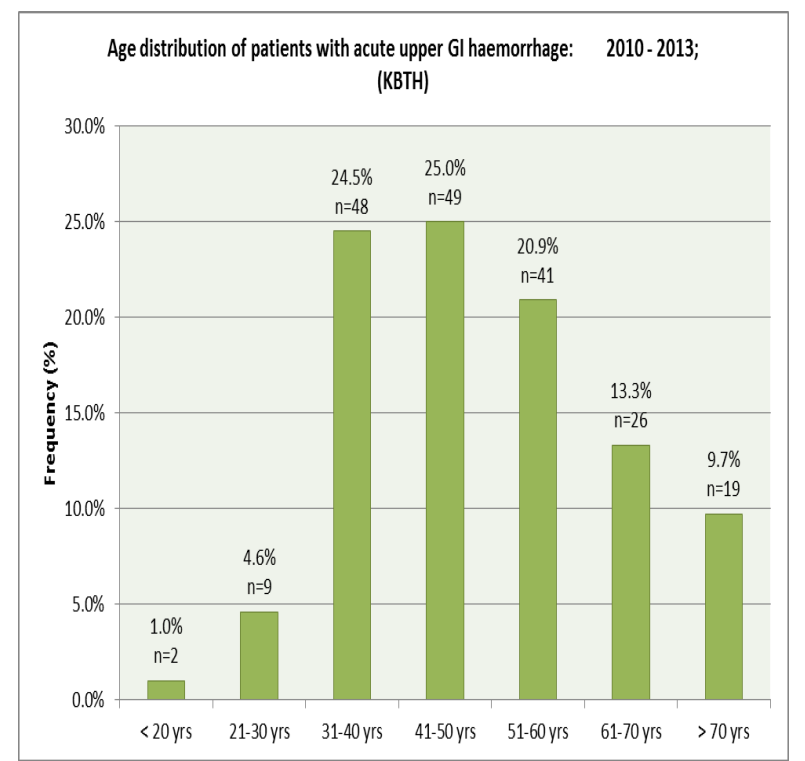

Figure 1 The Age distribution of patients with Upper GI Bleeding

Figure 2 shows mortalities following AUGIB in relation to duration of hospital stay. Outcomes following AUGIB were dismal with some $38 \%(n=75)$ of fatalities occurring within the first 24 hours and a further $16.3 \%(n=32)$ in the subsequent 24 hours.

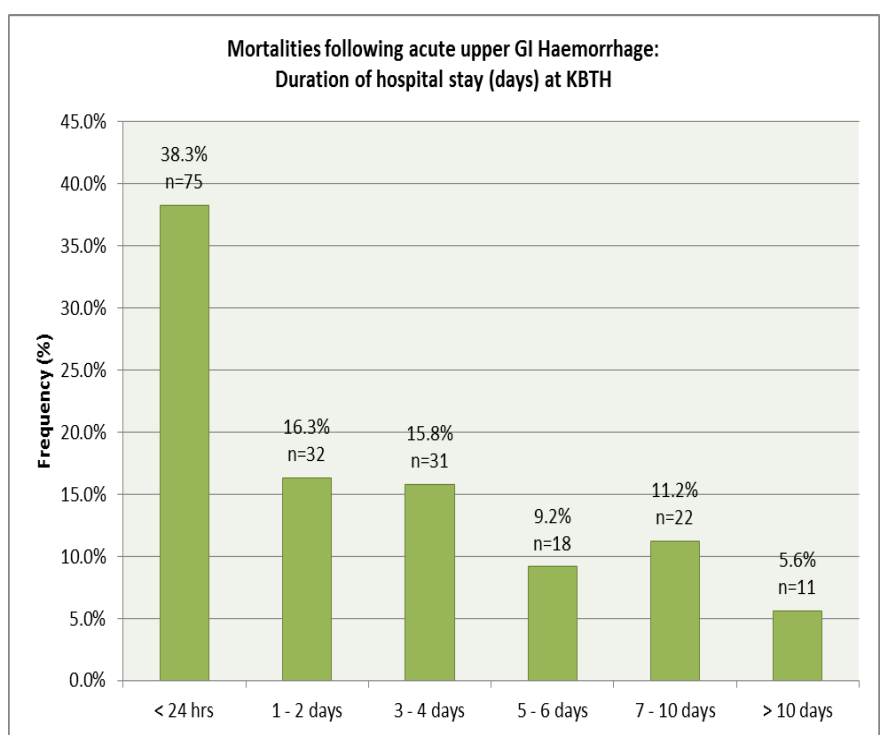

Figure 2 Duration of hospital stay and Mortality following Acute Upper GI Bleeding

A cross-sectional review of endo-therapy for GOVs at the Endoscopy Unit, KBTH, demonstrated that the mainstay of treatment was injection-sclerotherapy, performed in 59 patients during the study period. Howev- er, variceal rubber-band ligation therapy was also used when available.

The sclerosant used was $5 \%$ ethanolamine oleate with 2-3 $(2.5 \mathrm{ml})$ peri-variceal injections per sclerotherapy cycle.

All patients had large GOVs, North Italian Endoscopic Classification (NIEC) (10), with multiple columns of distended varices. Thirty-two percent $(32 \%, n=19)$ of patients had dysphagia following injection sclerotherapy with a mean duration of 5.73 days; however no oesophageal strictures were recorded following endoscopy. Fifty-nine percent $(59 \%, n=35)$ of patients had experienced post-procedure pain in the chest or abdomen which required either antacids or oral analgesia with paracetamol or tramadol.

\section{DISCUSSION}

Bleeding from the upper gastrointestinal tract is associated with significant morbidity and mortality. In the UK it accounts for $8 \%$ of emergency hospital admissions ${ }^{11}$ with an incidence of $47-116 / 100,000$ populations. $^{12}$ The aetiology of AUGIB and relationship with mortality varies across populations. A UK population-based multi-centre, prospective survey reviewed over 4,000 cases of AUGIB across 74 hospitals showing prevalence for GOVs, DU and GU as $4 \%, 18.6 \%$ and $12.3 \%$ respectively. ${ }^{13}$

Mortality was however inversely related to prevalence; $(23 \%)$ in variceal and $(12 \%)$ in peptic ulcer disease patients ${ }^{13}$. By contrast, in our study, GOVs were identified in $22 \%$ of cases in comparison to DU, $17.0 \%$; and $\mathrm{GU}, 13.2 \%$. It was second to peptic ulcer disease, which had an overall prevalence of $30.2 \%$ $(\mathrm{DU}+\mathrm{GU})$. This pattern was also demonstrated in a review of the clinical epidemiology of AUGIB in KBTH where there was a significant prevalence of bleeding GOVs amounting to $30 \%$, almost equal to that for DU and GU cases which was 39.1\%. ${ }^{14}$ Gastrooesophageal varices also remained the dominant cause of death from AUGIB ranging from $46 \%$ in 2010 to $76 \%$ in 2013 in our review. Similarly, a review at the Kenyatta National Hospital ${ }^{15}$ demonstrated that GOVs were the commonest cause of AUGIB and were also responsible for the majority of its case fatalities.

However, this trend in GOV prevalence has not always been evident in previous endoscopic series at KBTH. A review of upper-GI endoscopies for AUGIB between 1981-1983 assessed 86 such patients with prevalence rates of GOV, 4.7\%; DU, 31.4\%; and GU, $8.1 \%{ }^{16}$. In 1982, Helicobacter pylori were discovered ${ }^{17}$ and the 1980 s saw the advent of proton-pump inhibitors (PPI) for the treatment of acid-peptic diseases. ${ }^{18}$ 
It is likely that the relative increase in GOVs and concomitant reduction in prevalence of DU and GU may reflect the significant rise in PPI use and $H$. pylori therapy in dyspeptic patients prior to endoscopy. A pattern therefore emerges which shows that variceal bleeding is a much more common cause of AUGIB in Ghana and Sub-Saharan Africa in comparison with developed countries. However, its attendant mortality remains high irrespective of geographic location.

What seems alarming in our study is the high case fatality in the first 24 hours of emergency admission in patients with AUGIB (38\%). Difficulties faced in resuscitation, availability of appropriate facilities such as Seng-staken Blakemore tubes, access to therapeutic endoscopy and delayed referral are likely reasons in the face of such a burden of disease at the Tertiary Centre.

Majority of sufferers were unfortunately relatively young, within the 30-50 year group, who are usually the backbone of the workforce and families in society. On the contrary, a similar sample population of patients with AUGIB in the UK was rather elderly, with a mean age of 66 years. $^{13}$ This may reflect the differing aetiologies geographically. In Ghana for instance there is a significant burden of Hepatitis B acquired predominantly in early life with a prevalence of approximately $12-15 \%{ }^{19}$ leading to cirrhotic liver disease and associated portal hypertension. ${ }^{20}$ Liver cirrhosis is the commonest liver disease causing death in Ghana. ${ }^{20}$

Prevalence of Hepatitis B in patients with cirrhotic liver disease was found to be $42.9 \%{ }^{20}$ Local data on other causes of liver injury such as alcohol and potentially hepatotoxic herbal medications are however lacking. In Europe, however, elderly patients tend to be more vulnerable to AUGIB particularly from peptic disease as a result of the higher incidence of multiple co-morbid conditions and use of non-steroidal antiinflammatory drugs (NSAIDs). ${ }^{2,21,22}$

Injection sclerotherapy was the dominant endoscopic modality for secondary prevention of variceal bleeding in comparison with band ligation, mainly as a result of cost and availability. Over the study period, morbidity from the use of regular injection sclerotherapy was evident albeit temporary, with $32 \%$ of patients having reported dysphagia lasting approximately 5 days and some $60 \%$ experiencing post-procedure pain requiring analgesia. Both sclerotherapy and ligation can effectively arrest active bleeding from GOVs. ${ }^{23,24}$

However, ligation is more-effective than sclerotherapy in decreasing the risk of re-bleeding from GOVs and can achieve variceal obliteration more rapidly with fewer complications such as ulceration, bleeding or oesophageal stricturing. ${ }^{23}$ In our study, the use of injection sclerotherapy was not associated with endoscopic stricture formation possibly as a result of the large varices encountered and therefore reduced para-variceal intimal injection and subsequent sclerosis.

In Ghana, bleeding GOVs are a significant cause of AUGIB, identified in some $22 \%$ of patients with a comparatively high mortality. Cases demonstrated a male preponderance with in-hospital mortality (in 24 hours) reflecting inadequate facilities to deal with this medical emergency. It is recommended that unstable patients are transfused with blood promptly, with platelets if counts are below $50 \times 10^{9} / \mathrm{ml}$ and fresh frozen plasma if INR above $1.5 .^{25}$

Diagnostic and therapeutic endoscopy (e.g. band ligation) is essential in all unstable patients after resuscitation and to all other patients with AUGIB within 24 hours. $^{25}$ Octreotide or terlipressin in addition to prophylactic antibiotics with gram-negative cover also reduce morbidity and mortality in acute variceal bleeding. ${ }^{25,26}$

It is therefore hoped that an orchestrated and strategic approach to care with endoscopic services equipped with all the necessary therapeutic interventions will be vital in improving the outcomes of patients with variceal bleeding in Ghana. Further study evaluating the impact of other potential causes of cirrhotic liver disease would be important as it gains prominence in Ghana.

\section{ACKNOWLEDGEMENTS}

We are grateful to Margaret Reynolds, Research Assistant, Department of Medicine, for her assistance in sampling and collating data in the Department of Medicine and the Endoscopy Unit, Korle-Bu Teaching Hospital. We thank the Department of Medicine and staff of the Endoscopy Unit, KBTH for their assistance and support during the study.

\section{REFERENCES}

1. Bager P, Dahlerup JF. Lack of follow-up of anaemia after discharge from an upper gastrointestinal bleeding centre. Danish medical journal. 2013; 60(3):A4583.

2. El-Tawil AM. Trends on gastrointestinal bleeding and mortality: where are we standing? World journal of gastroenterology: WJG. 2012; 18(11):11548.

3. Sarin SK, Kumar A, Angus PW, Baijal SS, Chawla YK, Dhiman RK, et al. Primary prophylaxis of 
gastroesophageal variceal bleeding: consensus recommendations of the Asian Pacific Association for the Study of the Liver. Hepatology international. 2008; 2(4):429-39.

4. Weinstein W M HCJ, Bosch J. Portal hypertension. Clinical Gastroenterology and Hepatology 2005:707-14.

5. D'Amico G, De Franchis R, Cooperative Study G. Upper digestive bleeding in cirrhosis. Posttherapeutic outcome and prognostic indicators. Hepatology. 2003;38(3):599-612.

6. Biecker E. Portal hypertension and gastrointestinal bleeding: diagnosis, prevention and management. World journal of gastroenterology 2013;19(31):5035-50.

7. Bosch J, Garcia-Pagan JC. Complications of cirrhosis. I. Portal hypertension. Journal of hepatology 2000;32(1 Suppl):141-56.

8. Wright AS, Rikkers LF. Current management of portal hypertension. Journal of gastrointestinal surgery 2005;9(7):992-1005.

9. Blankson A WE, Gyasi RK, Adjei A, Tettey Y. Sero-Prevalence of Hepatitis B and C Viruses in Cirrhosis of the Liver in Accra, Ghana. Ghana Med J. 2005;39(4):132-7.

10. Sarin SK, Bhatia V., Wu J.C., Sung J. Upper Gastro-Intestinal Bleeding. In: Gastroenterological Endoscopy. 2011;3rd Ed:577-8.

11. Higham J, Kang JY, Majeed A. Recent trends in admissions and mortality due to peptic ulcer in England: increasing frequency of haemorrhage among older subjects. Gut 2002;50(4):460-4.

12. Dallal HJ, Palmer, K.R. Upper GI Haemorrhage. In: Logan, R PH et al ABC of the Upper GI Tract London: BMJ Books. 2002.

13. Rockall TA, Logan RF, Devlin HB, Northfield TC. Incidence of and mortality from acute upper gastrointestinal haemorrhage in the United Kingdom. Steering Committee and members of the National Audit of Acute Upper Gastrointestinal Haemorrhage. BMJ. 1995;311(6999):222-6.

14. Archampong E.Q., Essuman V.A., Dakubo J.C.B., Clegg-Lamptey J.N. Management of Upper Gastrointestinal Bleeding. In: Current Challenges with their Evolving Solutions in Surgical Practice in West Africa: A Reader University of Ghana 2013; 1:88-103.

15. Lodenyo H, Okoth, F. An observational study of oesophageal variceal endoscopic injection sclerotherapy in patients with portal hypertension seen at the Centre for Clinical Research, Kenya Medical Research Institute. Afr J Health Sci. 2007; 14:2168.
16. Nkrumah KN, Archampong E.Q., QuarteyPapafio, J.B., Ofori-Adjei D., Nakajima A. Acute Upper Gastro-Intestinal Haemorrhage in Ghanaians - An Endoscopic Review at the Korle-Bu Teaching Hospital. Ghana Med J. 1994;27(28):536-42.

17. Marshall BJ, Warren JR. Unidentified curved bacilli in the stomach of patients with gastritis and peptic ulceration. Lancet. 1984;1(8390):1311-5.

18. Sachs G, Shin JM, Vagin O, Lambrecht N, Yakubov I, Munson K. The gastric H,K ATPase as a drug target: past, present, and future. Journal of clinical gastroenterology 2007;41 Suppl 2:S22642.

19. Geretti AM, Patel M, Sarfo FS, Chadwick D, Verheyen J, Fraune M, et al. Detection of highly prevalent hepatitis B virus coinfection among HIVseropositive persons in Ghana. Journal of clinical microbiology 2010;48(9):3223-30.

20. Blankson A WEK, Gyasi R K, Adjei A, Tettey Y. Sero-Prevalence of Hepatitis B and C Viruses in Cirrhosis of the Liver in Accra, Ghana. Ghana Med J 2005;39((4)):132-7.

21. Lanas A, Garcia-Rodriguez LA, Polo-Tomas M, Ponce M, Alonso-Abreu I, Perez-Aisa MA, et al. Time trends and impact of upper and lower gastrointestinal bleeding and perforation in clinical practice. The American journal of gastroenterology 2009;104(7):1633-41.

22. Lanas A. Editorial: Upper GI bleeding-associated mortality: challenges to improving a resistant outcome. The American journal of gastroenterology 2010;105(1):90-2.

23. Lo GH, Lai KH, Cheng JS, Hwu JH, Chang CF, Chen SM, et al. A prospective, randomized trial of sclerotherapy versus ligation in the management of bleeding esophageal varices. Hepatology 1995;22(2):466-71.

24. Pereira SP, Wilkinson ML. Prospective randomized trial of endoscopic sclerotherapy versus variceal band ligation for esophageal varices: influence on gastropathy, gastric varices and variceal recurrence. Gastrointestinal endoscopy 1997;46(4):384-5.

25. (NICE) NIfHaCE. Acute Upper Gastro-Intestinal Bleeding. http://publicationsniceorguk/acuteupper-gastrointestinal-bleeding-managementcg141. June 2012.

26. Ioannou GN, Doust J, Rockey DC. Systematic review: terlipressin in acute oesophageal variceal haemorrhage. Alimentary pharmacology \& therapeutics.2003;17(1):53-64. 